DOI: 10.1136/annrheumdis-2019-eular.4236

\section{AB0234 AN INTEGRATED PROTEOMICS AND ANTIBODY ANALYSIS OF THE U-ACT-EARLY TRIAL TO IDENTIFY MARKERS OF TREATMENT RESPONSE AND DISEASE PROGRESSION IN EARLY RHEUMATOID ARTHRITIS}

Francesco Brizzi ${ }^{1}$, Suleiman A. Khan ${ }^{2}$, Marco Prunotto ${ }^{1}$, Jenny Devenport ${ }^{3}$, Attila Pethoe-Schramm ${ }^{1}$, Michelle Borm ${ }^{4}$, Johannes Wj Bijlsma ${ }^{5}$, Johannes W. G. Jacobs ${ }^{5}$, Floris Lafeber ${ }^{5}$, Paco Welsing ${ }^{5}$, Tero Aittokallio ${ }^{2,6}$, Antoine Soubret ${ }^{1}$. ${ }^{1}$ F. Hoffmann-La Roche, Basel, Switzerland; ${ }^{2}$ Institute for Molecular Medicine Finland FIMM, University of Helsinki, Helsinki, Finland; ${ }^{3}$ Genentech, Inc., South San Francisco, United States of America; ${ }^{4}$ Roche Nederland BV, Woerden, Netherlands; ${ }^{5}$ University Medical Center Utrecht, Utrecht, Netherlands; ${ }^{6}$ University of Turku, Department of Mathematics and Statistics, Turku, Finland

Background: Predictive markers of treatment response in rheumatoid arthritis (RA) are necessary to stratify patients to devise personalised treatment strategies. The U-Act-Early trial [1], in which disease-modifying antirheumatic drugs (DMARD) naïve early RA patients initiated either methotrexate (MTX) or tocilizumab (TCZ) in monotherapy or in combination, offers a unique opportunity to assess the capacity of many baseline -omics markers (i.e. genomics, transcriptomics, proteomics, metabolomics, auto-antibodies) to predict clinical outcomes.

Objectives: To identify predictive markers of treatment response by developing a statistical machine learning methodology for correlating baseline clinical and -omics variables with clinical response data over time.

Methods: We developed a multi-omics approach that combines longitudinal statistical modelling of clinical data with Bayesian machine learning variable selection to identify the most predictive markers of treatment response. The time course of clinical outcomes was fit using a non-linear mixed longitudinal model, that characterise each patient of the U-Act-Early trial $(n=317)$ by three parameters: a baseline value, an asymptotic value and a velocity (describing the speed at which the asymptote is reached). Supervised machine learning methods (such as Bayesian sparse regression as well as tree-based algorithms) were subsequently employed to find predictive markers for each of the longitudinal model parameters. We specifically focused on 85 auto-inflammatory proteins and 450 auto-antibodies markers, including 41 citrullinated-peptides antigens. The analysis was repeated for the three treatment-arms since DAS28-subscores response markers may vary across treatment strategies and clinical outcomes. We finally investigated whether predictions are enhanced by incorporating known networks of protein-protein interactions, rather than considering the predictive power of each protein independently.

Results: Preliminary results are that some proteins (e.g. SCD14, VEGF) were moderately predictive of the disease status at baseline. Specifically, the available inflammatory proteins predicted baseline DAS28, swollen and tender joints and ESR with $R^{2}$ values of $0.25,0.32,0.16$ and 0 respectively. Surprisingly, these proteins did not improve the prediction of treatment-response (i.e. the velocity and asymptotic parameters) after controlling for the baseline disease status. Protein networks marginally improved prediction when compared to prediction of individual proteins. Citrullinated peptide antigens did not appear predictive of the baseline disease status nor the treatment response.

Conclusion: In our initial investigation inflammatory proteins, especially when considered as part of interaction networks, appear to be predictive of the baseline status of the disease but not of treatment-response. The most predictive marker of treatment response appears to be disease activity at baseline.

\section{REFERENCES:}

[1] Bijlsma JWJ, et al. Lancet. 2016;388:343-55.

Acknowledgement This study was funded by F. Hoffmann-LaRoche Disclosure of Interests: Francesco Brizzi Employee of: F. Hoffmann-La Roche, Suleiman A. Khan: None declared, Marco Prunotto Employee of: F. Hoffmann-La Roche, Jenny Devenport Employee of: F. Hoffmann-La Roche, Attila Pethoe-Schramm Shareholder of: F. Hoffmann-La Roche, Employee of: F. Hoffmann-La Roche, Michelle Borm Employee of: An employee of Roche Nederland BV, Johannes WJ Bijlsma Grant/research support from: The department of the author who included patients (JWJB) in the U-Act-Early trial received reimbursements from Roche Nederland BV. JWJB reported grants and fees from Roche, AbbVie, Bristol-Myers Squibb, Merck Sharp \& Dohme, Pfizer, and UCB University Medical
Center Utrecht, Utrecht University, Consultant for: SUN Pharma, Speakers bureau: Lilly, Roche, Johannes W. G. Jacobs Grant/research support from: Roche, Consultant for: Roche, Floris Lafeber Shareholder of: ArthroSave, Grant/research support from: FOREUM; Dutch Arthritis Society, Paco Welsing: None declared, Tero Aittokallio: None declared, Antoine Soubret Employee of: F. Hoffmann-La Roche DOI: 10.1136/annrheumdis-2019-eular.5049

\section{AB0235 THE LIFETIME RISK OF KNEE AND HIP REPLACEMENT FOLLOWING A DIAGNOSIS OF RHEUMATOID ARTHRITIS: FINDINGS FROM ROUTINELY COLLECTED DATA FROM ENGLAND}

Edward Burn ${ }^{1}$, Christopher Edwards ${ }^{1,2}$, David W. Murray ${ }^{1}$, Alan Silman ${ }^{1}$, Cyrus Cooper ${ }^{1,3}$, Nigel Arden $^{1,3}$, Rafael Pinedo-Villanueva ${ }^{1}$, Daniel PrietoAlhambra $1,4 .{ }^{1}$ NDORMS, University of Oxford, Oxford, United Kingdom; ${ }^{2} \mathrm{NIHR}$ Wellcome Trust Clinical Research Facility, University Hospital Southampton, Southampton, United Kingdom; ${ }^{3} \mathrm{MRC}$ Lifecourse Epidemiology Unit, Southampton University, Southampton, United Kingdom; ${ }^{4}$ GREMPAL Research Group, Idiap Jordi Gol and CIBERFes, Universitat Autonoma de Barcelona and Instituto de Salud Carlos III, Barcelona, Spain

Background: Understanding the lifetime risk of knee and hip replacement following a diagnosis of rheumatoid arthritis (RA) would help provide patients and clinicians with an indication of long-term prognosis and future health care utilisation.

Objectives: To estimate the lifetime risk of knee and hip replacement following a diagnosis of RA.

Methods: Routinely collected data from the English NHS was used to inform the analysis. Diagnosis of RA was identified using primary care records, with knee and hip replacement observed in linked hospital records. Parametric survival models were fitted for up to 15 years of follow up, with age, sex, Charlson comorbidity score, socioeconomic status, $\mathrm{BMI}$, and smoking status included as explanatory variables. A decision model was used to combine and extrapolate survival models to estimate lifetime risk. Lifetime risk was estimated for individuals with average characteristics (median for continuous variables and mode for categorical ones). The partial effect of explanatory factors on lifetime risk was assessed by re-running models with participant profiles varying in the explanatory factor of interest, while holding other characteristics constant at their average.

Results: 13,961 study participants with a diagnosis of RA were included. Lifetime risk of knee replacement and hip replacement was estimated to be $22 \%(95 \% \mathrm{Cl} 16 \%$ to $29 \%)$ and $17 \%(11 \%$ to $26 \%)$ following a diagnosis of RA for the average patient profile (non-smoking women aged 64 with no other comorbidities, BMl of 27 and in the top socioeconomic quintile). Risks were higher for younger patients, but broadly similar for men and women. Comorbidities, socioeconomic status, BMI, and smoking status had relatively little impact on lifetime risk.

Conclusion: This study has estimated lifetime risk of knee and hip replacement following a diagnosis of RA, allowing for a better understanding of long-term prognosis and healthcare resource use. These risks are more than double those of the general UK population.

Disclosure of Interests: Edward Burn: None declared, Christopher Edwards Grant/research support from: Abbvie, BMS, Biogen, Celgene, Fresenius, Janssen, Lilly, Mundipharma, Pfizer, MSD, Novartis, Roche, Samsung, Sanofi, UCB, Consultant for: Abbvie, BMS, Biogen, Celgene, Fresenius, Janssen, Lilly, Mundipharma, Pfizer, MSD, Novartis, Roche, Samsung, Sanofi, UCB, Speakers bureau: Abbvie, BMS, Biogen, Celgene, Fresenius, Janssen, Lilly, Mundipharma, Pfizer, MSD, Novartis, Roche, Samsung, Sanofi, UCB, David W Murray Grant/research support from: Grants from Zimmer Biomet, Consultant for: Personal fees from Zimmer Biomet, Alan Silman: None declared, Cyrus Cooper Consultant for: Per sonal fees from Alliance for Better Bone Health, Amgen, Eli Lilly, GSK, Medtronic, Merck, Novartis, Pfizer, Roche, Servier, Takeda and UCB. Nigel Arden Grant/research support from: Grants from BIOIBERICA, and from MERCK., Consultant for: Personal fees from Flexion, from Regeneron, from Freshfields Bruckhaus Deringer, outside the submitted work. Rafael Pinedo-Villanueva: None declared, Daniel Prieto-Alhambra Grant/ research support from: Grants from Amgen, UCB Biopharma and Servie outside the submitted work, Consultant for: UCB Biopharma, Speakers bureau: Amgen

DOI: 10.1136/annrheumdis-2019-eular.2432 